OPEN ACCESS

Edited by:

Li Zhou,

Institute of Hydrobiology (CAS), China

Reviewed by:

Zhiyi Bai,

Shanghai Ocean University, China Mukunda Goswami, Central Institute of Fisheries Education (ICAR), India

*Correspondence: Yang Yu yuyang@qdio.ac.cn

Fuhua Li

fhli@qdio.ac.cn

Specialty section: This article was submitted to Livestock Genomics,

a section of the journal

Frontiers in Genetics

Received: 08 September 2021 Accepted: 01 November 2021 Published: 26 November 2021

Citation:

Zhang Q, Yu Y, Luo Z, Xiang J and Li F (2021) Comparison of Gene Expression Between Resistant and Susceptible Families Against VP $P_{A H P N D}$ and Identification of Biomarkers Used

for Resistance Evaluation in

Litopenaeus vannamei.

Front. Genet. 12:772442.

doi: 10.3389/fgene.2021.772442

\section{Comparison of Gene Expression Between Resistant and Susceptible Families Against VP AHPND and Identification of Biomarkers Used for Resistance Evaluation in Litopenaeus vannamei}

\author{
Qian Zhang ${ }^{1,2}$, Yang $Y u^{1,3 *}$, Zheng $L u 0^{1,2}$, Jianhai Xiang ${ }^{1,3}$ and Fuhua $L i^{1,3,4,5 *}$ \\ ${ }^{1}$ Key Laboratory of Experimental Marine Biology, Institute of Oceanology, Chinese Academy of Sciences, Qingdao, China, \\ ${ }^{2}$ University of Chinese Academy of Sciences, Beijing, China, ${ }^{3}$ Laboratory for Marine Biology and Biotechnology, Qingdao National \\ Laboratory for Marine Science and Technology, Qingdao, China, ${ }^{4}$ Center for Ocean Mega-Science, Chinese Academy of \\ Sciences, Qingdao, China, ${ }^{5}$ The Innovation of Seed Design, Chinese Academy of Sciences, Wuhan, China
}

Acute hepatopancreatic necrosis disease (AHPND) has caused a heavy loss to shrimp aquaculture since its outbreak. Vibrio parahaemolyticus $\left(\mathrm{VP}_{\mathrm{AHPND}}\right)$ is regarded as one of the main pathogens that caused AHPND in the Pacific white shrimp Litopenaeus vannamei. In order to learn more about the mechanism of resistance to AHPND, the resistant and susceptible shrimp families were obtained through genetic breeding, and comparative transcriptome approach was used to analyze the gene expression patterns between resistant and susceptible families. A total of 95 families were subjected to $\mathrm{VP}_{\mathrm{AHPND}}$ challenge test, and significant variations in the resistance of these families were observed. Three pairs of resistant and susceptible families were selected for transcriptome sequencing. A total of 489 differentially expressed genes (DEGs) that presented in at least two pairwise comparisons were screened, including 196 DEGs highly expressed in the susceptible families and 293 DEGs in the resistant families. Among these DEGs, 16 genes demonstrated significant difference in all three pairwise comparisons. Gene set enrichment analysis (GSEA) of all 27,331 expressed genes indicated that some energy metabolism processes were enriched in the resistant families, while signal transduction and immune system were enriched in the susceptible families. A total of 32 DEGs were further confirmed in the offspring of the detected families, among which 19 genes were successfully verified. The identified genes in this study will be useful for clarifying the genetic mechanism of shrimp resistance against Vibrio and will further provide molecular markers for evaluating the disease resistance of shrimp in the breeding program.

Keywords: gene expression, disease resistance, molecular marker, Vibrio parahaemolyticus, Litopenaeus vannamei 


\section{INTRODUCTION}

Litopenaeus vannamei is a commercially important aquaculture species, making up about $85 \%$ of total shrimp production in China (Qin et al., 2018). However, the shrimp aquaculture industry is continuously affected by the outbreak of viral and bacterial diseases, which have caused mass mortality and considerable economic losses. Vibrio parahaemolyticus carrying the PirA and PirB toxin genes in its plasmid $\left(\mathrm{VP}_{\mathrm{AHPND}}\right)$ is one of the most destructive pathogens in shrimp aquaculture, causing acute hepatopancreatic necrosis disease (AHPND) or early mortality syndrome (EMS) in L. vannamei (Lee et al., 2015). VP $\mathrm{VPH}_{\text {AHPD }}$ also caused AHPND in Penaeus monodon and Exopalaemon carinicauda (Soonthornchai et al., 2016; Ge et al., 2018). Therefore, prevention and control of AHPND are urgently needed in shrimp aquaculture.

Genetic selective breeding of disease resistance broodstock is a feasible and sustainable approach for the disease control. It has been proved to be efficient in controlling Taura syndrome virus (TSV). A disease-resistant line of L. vannamei against TSV had been established, and $18.4 \%$ increase in survival rate against TSV infection was obtained after one generation selection (Argue et al., 2002). For the selection of White spot syndrome virus (WSSV) resistance in L. vannamei, it showed that the average survival rates of generations G2 to G5 were $5.57 \%, 7.78 \%, 9.52 \%$, and $13.79 \%$, respectively (Huang et al., 2012). After three successive generation selection, the survival rates of E. carinicauda to $\mathrm{VP}_{\mathrm{AHPND}}$ increased from $26.67 \%$ to 36.67\% (Ge et al., 2018).

With the development of molecular biology, genomics approach offers a new possibility for accelerating the genetic selection process (Zhang X. et al., 2019). Identification of the major genes associated with disease resistance is the first step for marker-assisted selection (MAS) or gene-assisted selection (GAS) (Arora et al., 2019). So far, several genes associated with disease resistance have been reported in aquatic animals. Polymorphism of $L v A L F$ and TRAF6 was reported to be associated with the resistance to WSSV in shrimp (Wang et al., 2011; Liu et al., 2014b; Liu et al., 2014a; Yu et al., 2017; Zhang Q. et al., 2019). A major quantitative trait locus (QTL) for the resistance of Atlantic salmon (Salmo salar) against infectious pancreatic necrosis (IPN) was discovered, which has been already applied in marker-assisted breeding of IPN-resistant fish (Houston et al., 2008; Moen et al., 2009; Woldemariam et al., 2020). However, knowledge about the resistance to AHPND in shrimp is still poorly understood. A comparison of individuals or families with significant phenotype difference by transcriptome sequencing is an efficient way for screening the trait-associated genes. Based on the transcriptome data, myosin, myosin heavy chain, and chitinase were proved to be related to growth performance in L. vannamei (Santos et al., 2018). Transcriptome comparison between the families with high growth rate and low growth rate also illustrated that the genes related to cuticle, chitin, and muscle proteins were upregulated exclusively in higher growth families (Santos et al., 2021). Besides, the gene profiles of the Vibrioresistant and Vibrio-susceptible Meretrix petechialis families were analyzed, and several genes such as Big-Def, CTL9, and Bax were identified as candidate resistance-associated genes (Jiang et al., 2017).

In our previous work, we have carried out systematic family selection for the resistance trait to $\mathrm{VP}_{\mathrm{AHPND}}$ in L. vannamei. A total of 95 families were subjected to $\mathrm{VP}_{\mathrm{AHPND}}$ challenge test, and the results showed that significant resistance variations existed between different families. In the present study, we selected three AHPND resistant and three susceptible families for transcriptome sequencing and explored the differentially expressed genes (DEGs) in resistant and susceptible families. Several genes related to the resistance of shrimp against AHPND were identified. These genes might be developed as effective molecular markers for evaluating the disease resistance of shrimp, which could facilitate molecular marker-assisted breeding of shrimp.

\section{MATERIALS AND METHODS}

\section{Selection Resistant and Susceptible Families Against Acute Hepatopancreatic Necrosis Disease}

The full-sib families of L. vannamei were produced and stocked separately in Hainan Grand Suntop Ocean Breeding Co., Ltd, Wenchang, China. In order to identify the resistance of AHPND, the shrimp families were challenged by $\mathrm{VP}_{\text {AHPND }}$ each year, and the families with high survival rate were mated to generate the next-generation families. In 2018, 95 full-sib families with an average body weight of $2.10 \mathrm{~g}$ were selected for evaluation of resistance. For the challenge experiment, $\mathrm{VP}_{\mathrm{AHPND}}$ was prepared according to the method described by Zhang Q. et al. (2019). About 100 healthy shrimp from each family were subjected to $\mathrm{VP}_{\mathrm{AHPND}}$ immersion infection. Before the experiment, the shrimp were kept in the aquarium at a temperature of $26^{\circ} \mathrm{C} \pm$ $1^{\circ} \mathrm{C}$ for 3 days to acclimate them to the culture conditions. Then, the $\mathrm{VP}_{\mathrm{AHPND}}$ were added to the aquarium to make the concentration of $\mathrm{VP}_{\mathrm{AHPND}}$ as $5 \times 10^{6} \mathrm{CFU} / \mathrm{ml}$. Then, the dead shrimp were checked every $2 \mathrm{~h}$. The mortality of each family was recorded for $72 \mathrm{~h}$. The survival rates of the tested families are presented in Figure 1.

Considering the survival rate, growth stage, and pedigree information, three resistant families (VR4013, VR3837, and VR4027) and three susceptible families (VS3868, VS3879, and VS3880) were selected for transcriptome sequencing. The survival rates of three resistant families VR4013 (1.96 \pm $0.21 \mathrm{~g}), \operatorname{VR} 3837(2.14 \pm 0.33 \mathrm{~g})$, and VR4027 (2.21 $\pm 0.26 \mathrm{~g})$ were $87 \%, 96 \%$, and $78 \%$, and those of three susceptible families VS3868 $(1.87 \pm 0.24 \mathrm{~g}), \operatorname{VS} 3879(2.09 \pm 0.28 \mathrm{~g})$, VS3880 (2.19 \pm $0.30 \mathrm{~g}$ ) were $4 \%, 9 \%$, and $13 \%$, respectively (Figure 1). Based on their pedigree information, the families VR4013 and VS3868 were genetically related; therefore, the DEGs were analyzed between VR4013 and VS3868 in order to avoid the effects of genetic difference as far as possible. According to information of the growth stage and pedigree information, DEGs were analyzed between VR3837 and VS3879, and VR4027 and VS3880, respectively. 


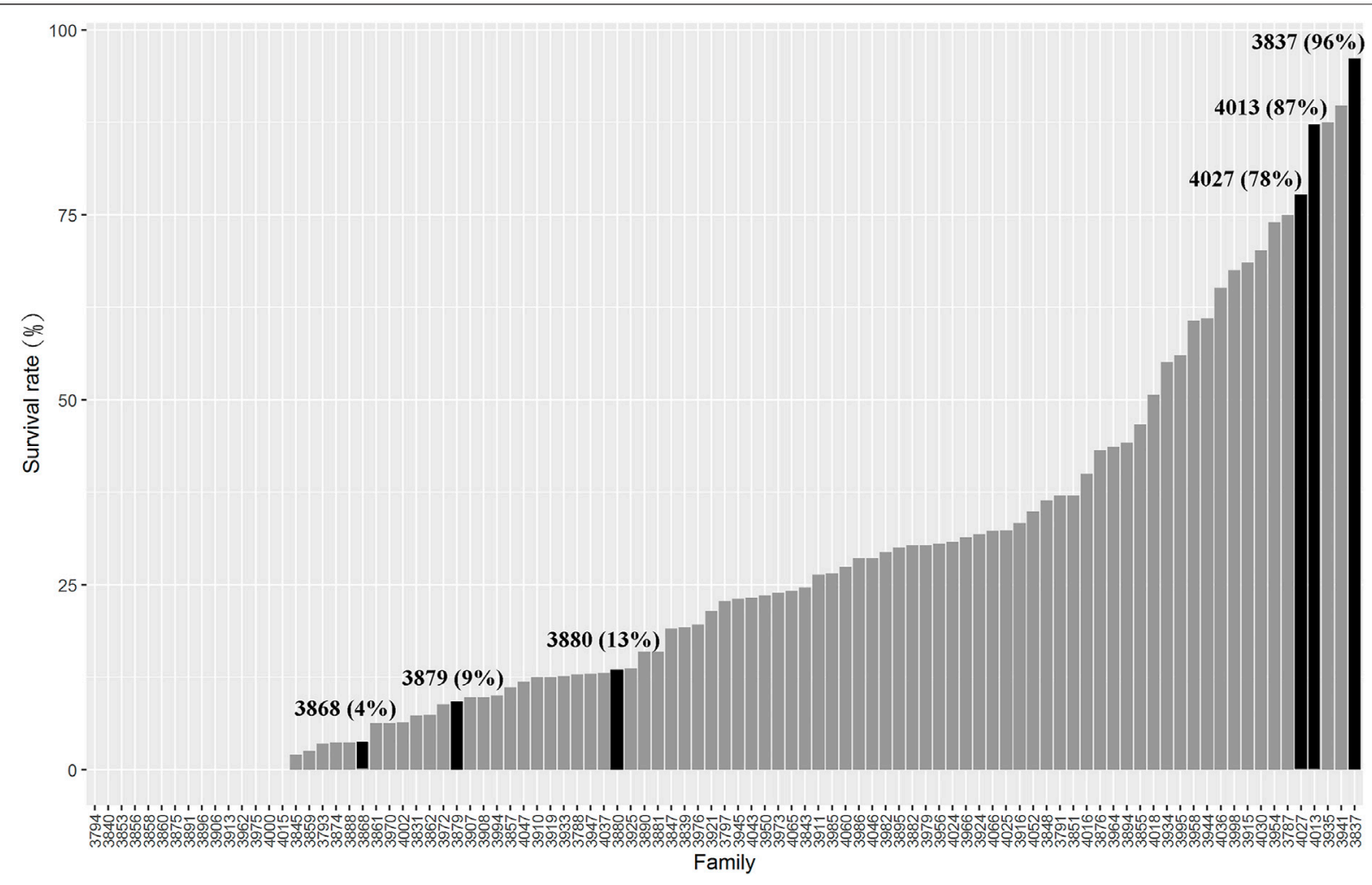

FIGURE 1 | Survival rate of different families challenged by $\mathrm{VP}_{\mathrm{AHPND}}$.

For each family, the cephalothoraxes of nine individuals were collected, and three individuals were mixed together as one sample, so each family contained three samples. All cephalothorax samples of the six families were rapidly frozen in liquid nitrogen and stored at $-80^{\circ} \mathrm{C}$ for further transcriptome sequencing.

\section{Total RNA Extraction, Library Construction, and Sequencing}

Total RNA of the cephalothorax was extracted using RNAiso Plus (Takara, Japan) according to the manufacturer's instructions. RNA purity and concentration were evaluated by electrophoresis on $1 \%$ agarose gel and quantified by NanoDrop 2000 spectrophotometer (Thermo Fisher Scientific, Waltham, MA, USA). Subsequently, mRNA was enriched by Oligo (dT) beads. Then the enriched mRNA was fragmented into short fragments using fragmentation buffer and reverse-transcribed into the first-strand cDNA with random primers. Secondstrand cDNA was synthesized by DNA polymerase I, RNase $\mathrm{H}$, dNTP, and buffer. Then the cDNA fragments were purified with QiaQuick PCR extraction kit, end repaired, poly (A) added, and ligated to Illumina sequencing adapters. The suitable fragments were selected by agarose gel electrophoresis. After PCR amplification, the libraries were sequenced using Illumina HiSeqTM 2,500 by Genedenovo Biotechnology Co., Ltd (Guangzhou, China).

\section{Bioinformatics Analysis}

Clean reads were obtained by removing reads containing adapters or more than $10 \%$ of unknown nucleotides $(\mathrm{N})$, low-quality reads, and rRNA from the raw data. Then the reads of each sample were mapped to reference genome (Zhang X. et al., 2019) by TopHat2 (version 2.0.3.12) (Kim et al., 2013). The reconstruction of transcripts was carried out with software Cufflinks (Trapnell et al., 2012). All reconstructed transcripts were aligned to reference genome, and novel genes were aligned to databases including National Center for Biotechnology Information (NCBI) nonredundant $(\mathrm{Nr})$ database, Swiss-Prot database, and Kyoto Encyclopedia of Genes and Genomes (KEGG) database to obtain protein functional annotation. DEGs between susceptible and resistant libraries were analyzed using the edgeR package (http://www.rproject.org/), with a fold change $\geq 2$ and a false discovery rate $(\mathrm{FDR})<0.05$. DEGs were then subjected to enrichment analysis of Gene Ontology (GO) functions and KEGG pathways, which were performed using the OmicShare tools (http://www.omicshare.com/tools).

\section{Gene Set Enrichment Analysis}

As a complement to the differential expression analyses, gene set enrichment analysis (GSEA) for all 27,331 expressed genes was performed by GSEA software v4.10 (https://www.gsea-msigdb. org/gsea/downloads.jsp) (Subramanian et al., 2005). The submitted gene list was ranked by gene expression value using Signal2Noise method. GSEA was performed with default 
TABLE 1 | Primer sequences and annealing temperature used for RT-qPCR

\begin{tabular}{|c|c|c|c|c|}
\hline Gene name & Forward primer $\left(5^{\prime}-3^{\prime}\right)$ & Reverse primer $\left(5^{\prime}\right.$ to $\left.3^{\prime}\right)$ & Product size (bp) & $\operatorname{Tm}\left({ }^{\circ} \mathrm{C}\right)$ \\
\hline ncbi_113822350 & ATTCCCTACAGTGACGACTA & GCCAAAAGATTCTCTCATGC & 132 & 51 \\
\hline ncbi_113828004 & GTTCCAAACCСTACTTGTCT & CTATGTCCAAAACGGAATGC & 106 & 51 \\
\hline XLOC_016514 & AGATGCTTCCCTGGATCAACC & TGGACTCTCCATTCCGATGTTC & 127 & 57 \\
\hline XLOC_016534 & TCGCCATGAAGAACTGGTCA & GCAAATTGAAGGCGTCAGCA & 96 & 56 \\
\hline ncbi_113824827 & TTGCGAGACAGACCAACCAG & CAGGTGCAATCTTCATCGCC & 133 & 55 \\
\hline ncbi_113816316 & TTCCTCCCGCAAGACAAG & GAGGGAGGGTTGGGTाা & 150 & 55 \\
\hline ncbi_113816839 & CTाTCGGGAGGGAGCGTAT & ACGGGAATAGTCCATCCAAGT & 162 & 56 \\
\hline ncbi_113810874 & ACCCGCTGTCCGCTCTACCA & TGTCCCAGCCGCAGCTCAAC & 118 & 64 \\
\hline ncbi_113805286 & GGGCAACTTACGGCTTCT & TTCGTGCCAATGGGTTC & 131 & 54 \\
\hline ncbi_113826200 & ATTGCAGCACCGTCTCCT & TCCCTCAGGCAGACTTCG & 91 & 57 \\
\hline XLOC_026751 & TCTTGTGCCTCGCTGTGG & GGTGATGTGCGTGATCTTCTT & 149 & 57 \\
\hline ncbi_113826199 & CTCACCGCTGCGAGGATT & TCCCTCAGGCAGACTTCG & 106 & 58 \\
\hline XLOC̄_023290 & TCTGCTGGTGATGATGGT & GTCATCGGGAGAACAACT & 142 & 52 \\
\hline ncbi_113808761 & CCGCAATGCTGTAGAAGGAC & CGGCGGTCAGAGTGGAGAT & 149 & 58 \\
\hline ncbi_113802520 & CTTCTTGCCGTGTाGCC & ACGATGCCGTCTCCTGTCT & 164 & 57 \\
\hline ncbi_113815780 & ACTCATAACCCACCGCCACT & TCGTCAGGGACCCAGCAA & 154 & 59 \\
\hline ncbi_1,13820830 & AAGCCGAACTTGGAGGACC & CGGATGAACTTACCGAAACG & 110 & 56 \\
\hline XLOC_016349 & CATCAAGCCCAAACCACC & TCTTCTCCAGCCAGCCACT & 104 & 58 \\
\hline XLOC_016348 & ATTGGACGCAAGGAGTATGG & CCTGGGCTGGTTGATGAG & 146 & 55 \\
\hline ncbi_1,13817858 & GAGGATGGGCTGAAATGTG & GTCCAGCAACTCTGAAGTATGA & 155 & 54 \\
\hline ncbi_113825958 & GGAACAGCAGACGGGAGTG & CAACGAAGCATTGGTGGC & 93 & 57 \\
\hline ncbi_113828431 & CCGTCACCAACACCCATAA & AGCAGCCACCCAAGGAAA & 150 & 56 \\
\hline ncbi_113807930 & GCTCGTCACCACAACCAT & CGAAGATGGGAGGCAGGT & 145 & 56 \\
\hline ncbi_113816695 & GAGCACCTCGCTाTCTGTाT & CATGACTTGGGTTCAGGTTA & 107 & 54 \\
\hline ncbi_113804592 & TCACGGAGTGCCGCTACGAT & TCCCTGTTGCGGATGTCCTG & 187 & 60 \\
\hline ncbi_113813557 & CTGCCAGTGGAACACGCTAT & GCGGTGCTAGGAACGTAACTAA & 186 & 57 \\
\hline ncbi_1,13817635 & ATGCTGACAAGGCGAATA & AAGAGTCAGACCCGCAAG & 159 & 52 \\
\hline ncbi_113830625 & CGTGAATCGCAGTCCCTA & GTGGTCGCTTCCTCTTCC & 100 & 55 \\
\hline ncbi_113807689 & GGCAGCCGCATCTTCATC & AGGGCGAAGCGGCGGTTGTT & 176 & 60 \\
\hline ncbi_1,13819349 & GTTCCATACCGCCGTTACCA & CGAGCAATITCGCTTACAACACTA & 119 & 55 \\
\hline ncbi_113806536 & GCACTTCCAAAGCCAACGA & GATCTCCTCGGAGTTGTAGCG & 119 & 57 \\
\hline ncbi_113802817 & CGTCGCTGGGCACAAGTA & AGCCGAAGTGTCCCGTTA & 167 & 57 \\
\hline ncbi_1,13817262 & AATGAGGCGGAGGAGCAG & CCTTCCAGGTGGCAGACAG & 92 & 58 \\
\hline ncbi_113821874 & TAAGAAGGTCCAGAGGCG & AACCCACAAGGCCATACA & 125 & 55 \\
\hline ncbi_113810465 & CGCTGGTGGGTGTCGTGAT & CCGCTTGGCTGCTGAGAT & 117 & 55 \\
\hline ncbi_113811111 & CCGAGGTCAACTACGAGG & ACGGGACTTGGTGGCTGGT & 106 & 55 \\
\hline ncbi_113816327 & АААСССААСССТСССТСТ & TCСTCCGTCTCCAACACC & 136 & 54 \\
\hline ncbi_113821801 & AAGGGCGTGGAAGGAATG & СТТСАТСТССТССТТСТССТТ & 187 & 56 \\
\hline $18 S$ & TATACGCTAGTGGAGCTGGAA & GGGGAGGTAGTGACGAAAAAT & 136 & 56 \\
\hline
\end{tabular}

algorithm as 1,000 permutations. GO gene sets $(10,192$ gene sets) and KEGG subset of canonical pathways (186 gene sets) were used as enrichment input, which were from Molecular Signatures database (MSigDB, https://www.gsea$\mathrm{msigdb}$.org/gsea/msigdb/collections.jsp). Nominal $p$-value < 0.05 and FDR $<0.25$ were considered as statistically significant.

\section{Evaluation on the Transcriptome Results by Real-Time Quantitative PCR}

Six genes were selected from each comparison group to evaluate the transcriptome sequencing result by RT-qPCR. About $1 \mathrm{mg}$ of total RNA was used to synthesize cDNA by PrimeScript ${ }^{\mathrm{TM}}$ RT reagent Kit with gDNA Eraser kit (Takara, Japan) according to the manufacturer's instructions. Gene-specific primers (Table 1) were designed using Primer 5, where 18S rRNA was used as the reference gene for RT-qPCR analysis. RT-
qPCR was conducted with the following conditions: denaturation at $94^{\circ} \mathrm{C}$ for $2 \mathrm{~min}, 40$ cycles of $94^{\circ} \mathrm{C}$ for $30 \mathrm{~s}$, annealing temperature for $20 \mathrm{~s}$, and $72^{\circ} \mathrm{C}$ for $30 \mathrm{~s}$. Each sample had four technical replicates. The specificity of the primer set was checked by melting curve analysis. The relative expression level was calculated with $2^{-\Delta \Delta C t}$ method (Livak and Schmittgen, 2001).

\section{Validation of Candidate Genes in Descendant Families}

In order to validate the identified DEGs, the descendant families of VS3868 and VR4013 were collected, and the DEGs were further validated in the descendant families by RT-qPCR. Family 4419 was produced by full-sib mating of family VS3868, and family 4253 was produced by full-sib mating of family VR4013. After being challenged by $\mathrm{VP}_{\mathrm{AHPND}}$, the survival rate of family 4253 was over 3.5 times higher than that of family 
TABLE 2 | Summary of transcriptome sequencing and assembly of the transcriptome from Litopenaeus vannamei.

\begin{tabular}{|c|c|c|c|c|}
\hline Sample & Raw reads & Clean reads & Percentage remained (\%) & Gene number \\
\hline VR4013-1 & $43,410,372$ & $42,125,030$ & 97.04 & 20,485 \\
\hline VR4013-2 & $57,672,500$ & $55,606,214$ & 96.42 & 20,677 \\
\hline VR4013-3 & $51,479,718$ & $49,787,374$ & 96.71 & 21,014 \\
\hline VR3837-1 & $38,338,506$ & $36,947,962$ & 96.37 & 19,828 \\
\hline VR3837-2 & $37,704,754$ & $36,467,784$ & 96.72 & 19,592 \\
\hline VR3837-3 & $47,019,886$ & $45,527,220$ & 96.83 & 20,211 \\
\hline VR4027-1 & $56,229,492$ & $54,118,212$ & 96.25 & 21,034 \\
\hline VR4027-2 & $39,363,752$ & $37,908,938$ & 96.30 & 20,832 \\
\hline VR4027-3 & $44,978,148$ & $43,327,096$ & 96.33 & 20,310 \\
\hline VS3868-1 & $42,426,736$ & $41,120,350$ & 96.92 & 20,912 \\
\hline VS3868-2 & $42,975,552$ & $41,754,004$ & 97.16 & 20,621 \\
\hline VS3868-3 & $41,490,144$ & $40,033,320$ & 96.49 & 20,573 \\
\hline VS3880-1 & $51,220,018$ & $49,705,886$ & 97.04 & 20,157 \\
\hline VS3880-2 & $45,819,944$ & $44,689,240$ & 97.53 & 20,289 \\
\hline VS3880-3 & $42,021,826$ & $40,653,124$ & 96.74 & 20,005 \\
\hline VS3879-1 & $49,914,612$ & $48,181,284$ & 96.53 & 20,111 \\
\hline VS3879-2 & 43,730,322 & $42,164,296$ & 96.42 & 19,971 \\
\hline VS3879-3 & $47,364,768$ & $45,863,282$ & 96.83 & 20,366 \\
\hline
\end{tabular}

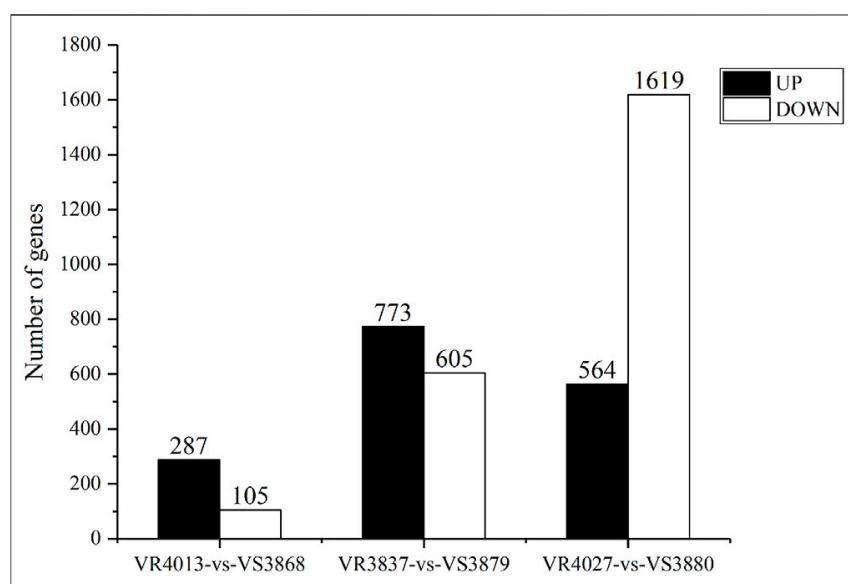

FIGURE 2 | The amount of differentially expressed genes (DEGs) between resistant and susceptible families. UP represents highly expressed genes in susceptible families. DOWN represents highly expressed genes in resistant families.

4419 (60 vs. 17\%). A total of 32 DEGs including the 16 DEGs shared in three pairwise comparisons and other 16 DEGs shared in two pairwise comparisons were selected, and they were verified in family 4419 and 4253 . The primers designed by Primer 5 are listed in Table 1. The RT-qPCR was performed as described in Evaluation on the Transcriptome Results by Real-Time Quantitative PCR. A statistically significant difference was indicated with ${ }^{*}(p<0.05)$ and ${ }^{\star *}(p<0.01)$ determined by $t$-test (SPSS Inc., Armonk, NY, USA).

\section{RESULTS}

\section{Transcriptome Sequencing Data}

An overview of sequencing and assembly of the L. vannamei transcriptome is shown in Table 2. A total of 823,161,050 raw reads were obtained, in which $416,197,128$ reads are for the resistant families and 406,963,922 reads for the susceptible families. The raw sequencing data were uploaded to the NCBI with the accession numbers SRR15533118-SRR15533135. After low-quality reads were filtered out, a total of $96.55 \%$ and $96.85 \%$ reads were retained for the resistant and susceptible groups, respectively. After the reads were mapped to the reference genome, a total of 27,331 annotated genes were obtained, of which $2,344(9.86 \%)$ were newly annotated genes.

\section{Identification of Differentially Expressed Genes Between Resistant and Susceptible Families}

To identify DEGs involved in $\mathrm{VP}_{\mathrm{AHPND}}$ resistance, we used FPKM value for comparing the expression levels between the resistant families and susceptible families. A total of 392 unigenes showed differential expression patterns between families VR4013 and VS3868, in which 287 unigenes were highly expressed in VS3868 and 105 unigenes were highly expressed in VR4013 (Figure 2). A total of 1,378 unigenes were differentially expressed between VR3837 and VS3879, including 773 unigenes highly expressed in VS3879 and 605 unigenes highly expressed in VR3837. A total of 2,183 unigenes were differentially expressed between VR4027 and VS3880, including 564 unigenes highly expressed in VS3880 and 1,619 unigenes highly expressed in VR4027. Six DEGs selected from each comparison group were validated by RT-qPCR. The results showed that all of them were consistent with the transcriptome data (Supplementary Figure S1).

In order to analyze the function of DEGs between resistant and susceptible families, GO functional enrichment analysis was performed. Interestingly, we found that GO terms of DEGs were very similar among three pairs of families, VR4013-vs.-VS3868, VR3837-vs.-VS3879, and VR4027-vs.-VS3880, which is shown in Figure 3A. In the biological process category, single- 

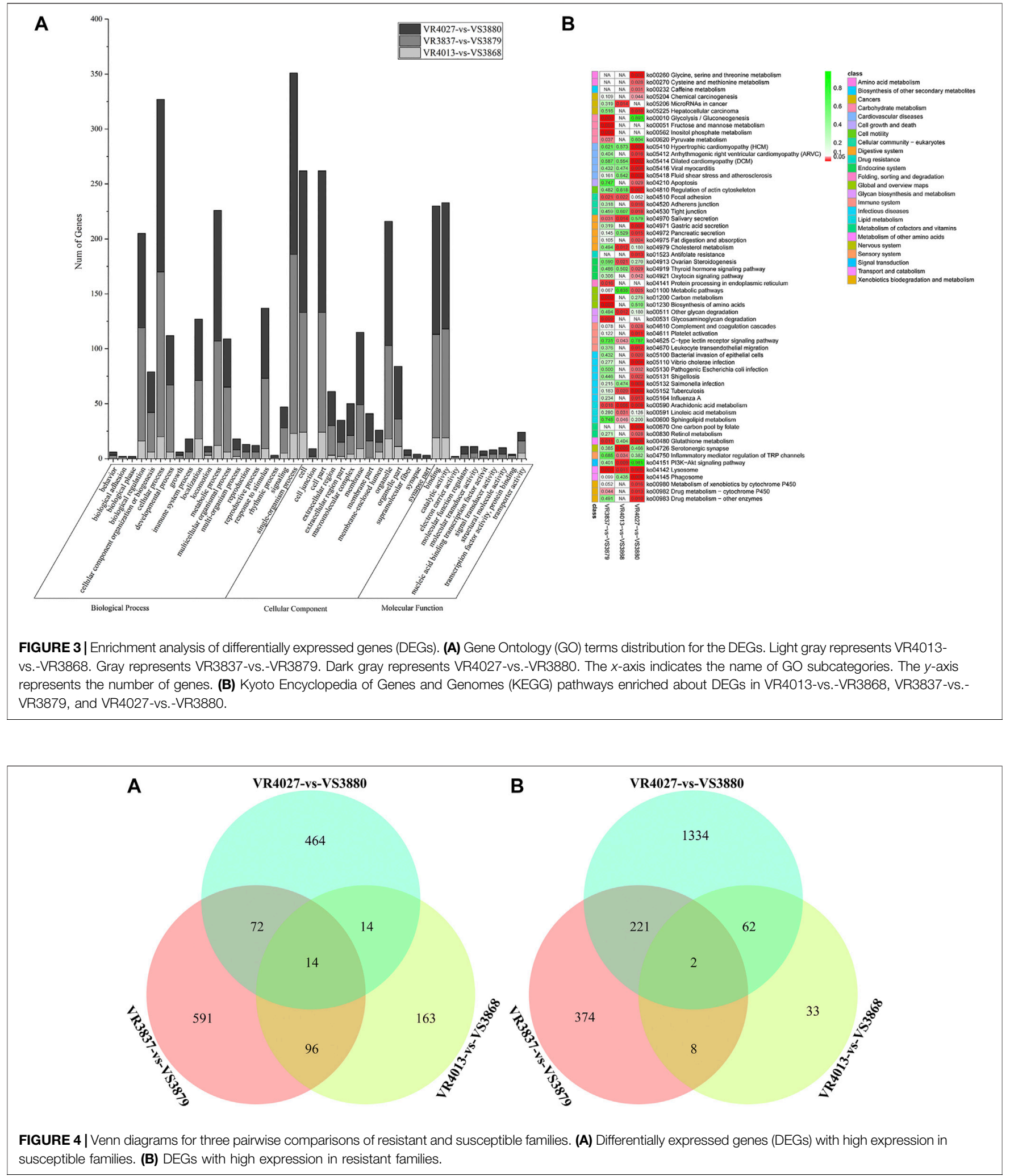

organism process was the most enriched subclasses, followed by cellular process. In the cellular component category, cell and cell part were the two most enriched subclasses. While in the molecular function category, catalytic activity and binding were the two most enriched subclasses. KEGG pathway enrichment analysis of three pairwise comparisons is 
TABLE 3 | Gene name, gene annotation, and the verification in hepatopancreas (Hep) and stomach (St) of 16 DEGs that were shared in three pairwise comparisons.

\begin{tabular}{|c|c|c|c|}
\hline \multirow[t]{2}{*}{ Gene name } & \multirow[t]{2}{*}{ Gene annotation } & \multicolumn{2}{|c|}{ Verification ( $p$-value) } \\
\hline & & Hep & St \\
\hline ncbi_113805286 & $N A^{a}$ & & $\sqrt{ }(0.001)$ \\
\hline ncbi_113810874 & Trophoblast glycoprotein & & $\sqrt{ }(0.024)$ \\
\hline ncbi_113826200 & NA & $\sqrt{ }(0.025)$ & \\
\hline XLOC_016514 & NA & & $\sqrt{ }(0.004)$ \\
\hline XLOC_016534 & NA & & \\
\hline ncbi_113822350 & SE-cephalotoxin-like & $\sqrt{ }(0.004)$ & $\sqrt{ }(0.01)$ \\
\hline ncbi_113824827 & Prolow-density lipoprotein receptor-related protein 1-like & $\sqrt{ }(0.018)$ & $\sqrt{ }(0.013)$ \\
\hline ncbi_113828004 & Peroxidasin-like protein & & $\sqrt{ }(0.02)$ \\
\hline ncbi_113819349 & Phosphoenolpyruvate carboxykinase, cytosolic & & \\
\hline ncbi_113807689 & Cytochrome P450 & & \\
\hline XLOC_026751 & NA & $\sqrt{ }(0.006)$ & $\sqrt{ }(0.014)$ \\
\hline ncbi_113826199 & Single insulin-like growth factor-binding domain protein-2 & $\sqrt{ }(0.003)$ & $\sqrt{ }(0.009)$ \\
\hline XLOC_023290 & NA & $\sqrt{ }(0.012)$ & \\
\hline ncbi_113825958 & Serpin B6-like isoform X2 & & \\
\hline ncbi_113816327 & NA & & \\
\hline ncbi_113816316 & NA & $\sqrt{ }(0.023)$ & \\
\hline
\end{tabular}

Note. The threshold for significance is $\mathrm{p}$-value $<0.05$.

DEGs, differentially expressed genes.

${ }^{a} N A$ indicates that the function of gene is unknown.

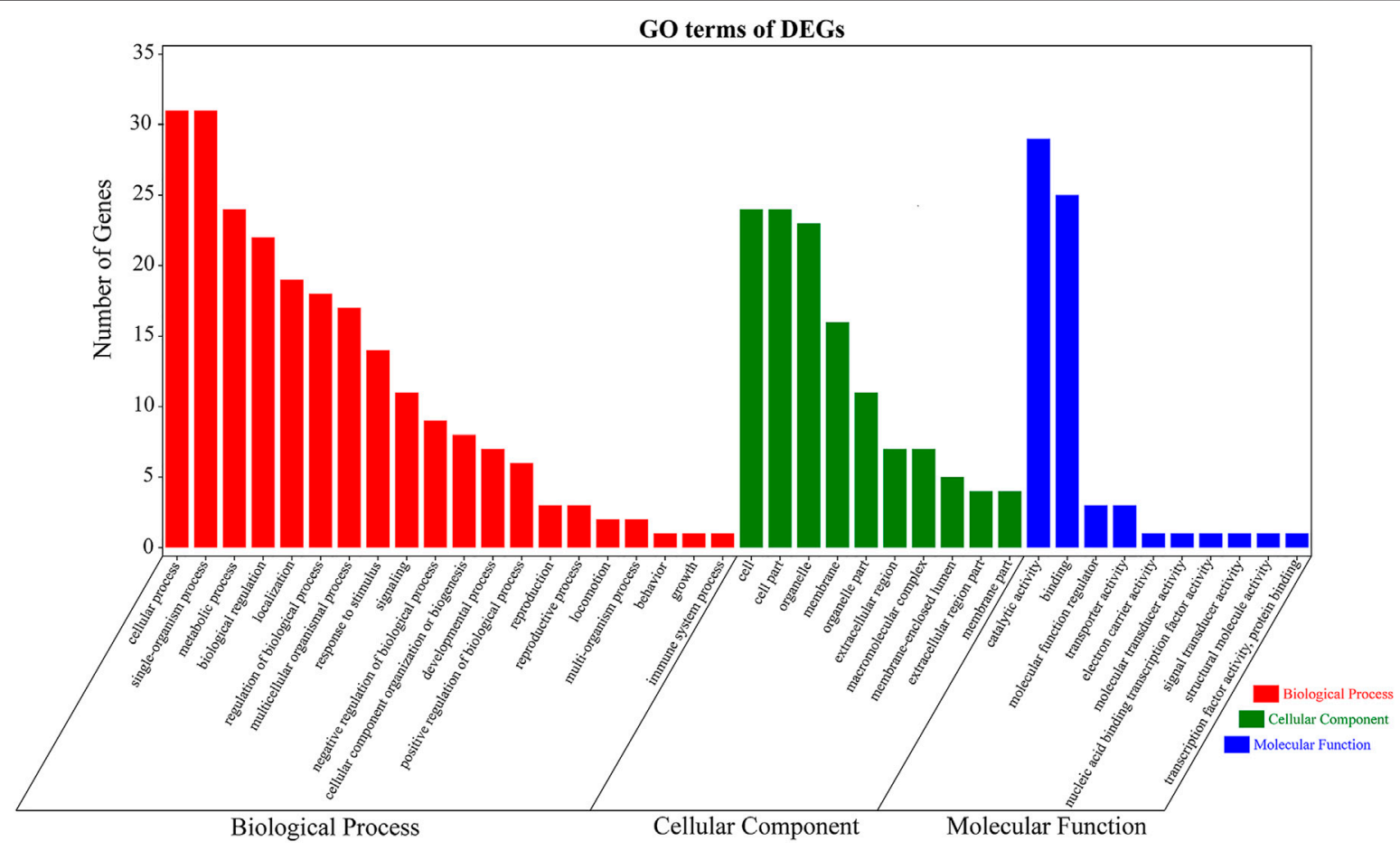

FIGURE 5|Gene Ontology (GO) terms of 489 differentially expressed genes (DEGs) for the transcriptomes of Litopenaeus vannamei. The $x$-axis indicates the name of GO subcategories. The $y$-axis indicates the number of genes. Red indicates biological process. Green indicates cellular component. Blue displays molecular function.

presented in Figure 3B. In VR4013-vs.-VS3868, "Serotonergic synapse," "Arachidonic acid metabolism," and "PI3K-Akt signaling pathway" were the three most enriched pathways, and "Arachidonic acid metabolism" was also included in top 20 of enrichment pathways in VR3837-vs.-VS3879 and VR4027vs.-VS3880 $(p<0.05)$.

\section{Differentially Expressed Genes Shared in More Than Two Pairwise Comparisons}

Venn diagrams for DEGs of the three pairwise comparisons are shown in Figure 4. A total of 489 DEGs were shared in at least two comparisons, including 196 DEGs highly expressed in the susceptible families and 293 DEGs highly expressed in resistant 


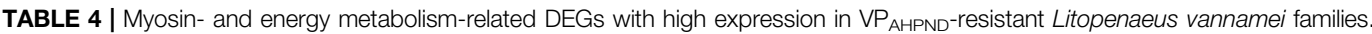

\begin{tabular}{|c|c|c|}
\hline Gene ID & Description & Species \\
\hline ncbi_113815780 & Myosin-16-like & Lepisosteus oculatus \\
\hline ncbi_113820830 & Myosin-16 isoform X2 & L. oculatus \\
\hline XLOC__031566 & Myosin heavy chain, muscle-like isoform $X 7$ & Hyalella azteca \\
\hline ncbi_113816531 & Myosin-7 & Chelonia mydas \\
\hline XLOC̄_016349 & Myosin heavy chain type 2 & L. vannamei \\
\hline XLOC_016348 & Myosin heavy chain type 2 & Penaeus monodon \\
\hline XLOC_016355 & Myosin heavy chain type $b$ & Marsupenaeus japonicus \\
\hline XLOC_015656 & Myosin heavy chain type b & M. japonicus \\
\hline ncbi_113802000 & Myosin heavy chain, partial & Rana catesbeiana \\
\hline XLOC_009141 & Actin 2 & Nilaparvata lugens \\
\hline ncbi_113815891 & Tryptase-like & Lates calcarifer \\
\hline ncbi_113822347 & Tryptase-2-like & Scleropages formosus \\
\hline ncbi_113813760 & Trypsin-like & Sinocyclocheilus rhinocerous \\
\hline ncbi_113809217 & Trypsin-2-like & S. rhinocerous \\
\hline ncbi_113814573 & Chymotrypsinogen A & Ovis aries musimon \\
\hline ncbi_113806996 & Serine protease 33 -like & Python bivittatus \\
\hline ncbi_113800311 & Serine protease 27 -like & L. calcarifer \\
\hline XLOC_005102 & Aminopeptidase $\mathrm{N}$-like & H. azteca \\
\hline ncbi_113815289 & Aminopeptidase $\mathrm{N}$ & Ornithorhynchus anatinus \\
\hline ncbi_113815282 & Aminopeptidase $\mathrm{N}$ & Octodon degus \\
\hline ncbi_113815313 & Aminopeptidase $\mathrm{N}$ & Ochotona princeps \\
\hline ncbi_113815275 & Aminopeptidase Ey-like & Xenopus laevis \\
\hline ncbi_113811393 & Aminopeptidase Ey-like & $X$. laevis \\
\hline ncbi_113804819 & Aminopeptidase Ey-like & $X$. laevis \\
\hline ncbi_113804804 & Aminopeptidase N, partial & Chlorocebus sabaeus \\
\hline XLOC_009027 & Cathepsin I, partial & L. vannamei \\
\hline ncbi_113807506 & Pancreatic lipase-related protein 2-like & Chaetura pelagica \\
\hline ncbi_113821709 & Phospholipase A2 & Pagrus major \\
\hline ncbi_113808761 & Alkaline phosphatase, tissue-nonspecific isozyme-like & S. rhinocerous \\
\hline ncbi_1,13820123 & Glyceraldehyde 3-phosphate dehydrogenase & Esox lucius \\
\hline ncbi_113802551 & Triosephosphate isomerase A & Astyanax mexicanus \\
\hline
\end{tabular}

Note. DEGs, differentially expressed genes.

families (Supplementary Table S1). A total of 16 DEGs were shared in three pairwise comparisons, among which the trophoblast glycoprotein, SE-cephalotoxin-like, peroxidasin-like protein, phosphoenolpyruvate carboxykinase, cytochrome P450, and serpin B6-like isoform X2 genes were identified (Table 3). In order to reduce the false positives, we considered these 489 DEGs as the candidate genes associated with the resistance of shrimp against to $\mathrm{VP}_{\mathrm{AHPND}}$.

These 489 DEGs were involved in various GO classifications. For the biological process-related genes, most were involved in "single-organism process," "cellular process," and "metabolic process." Most of the cellular component-related genes were associated with "cell," "cell part," and "organelle." And "catalytic activity" and "binding" in the molecular function ontology were the major enriched terms (Figure 5). The results were consistent with GO functional enrichment analysis in Figure 3A.

\section{Differentially Expressed Genes with High Expression in Resistant Families}

There were 293 DEGs highly expressed in resistant families. A total of nine genes annotated as myosin were highly expressed, and the other genes were involved in energy metabolism such as trypsin, chymotrypsinogen A (ChyA), pancreatic lipase (PL), serine protease (SP), aminopeptidase, and phospholipase (Table 4). There were two genes encoding glyceraldehyde 3-phosphate dehydrogenase (GAPDH) and triosephosphate isomerase (TPI), which were involved in the glycolysis pathway (Eanes, 2011).

\section{Differentially Expressed Genes with High Expression in Susceptible Families}

A total of 196 DEGs highly expressed in the susceptible families were discovered. According to annotation and function, some immune-related genes were observed to be upregulated in the susceptible families (Table 5). A total of five genes related to the prophenoloxidase (proPO) system, including C-type lectin, SP, and SP inhibitors (serpin and $\alpha$-2-macroglobulin) showed high expression in the susceptible families. Several genes are related to metabolic process, including $\mathrm{DBH}$-like monooxygenase protein 1, cytochrome b5, and cytochrome P450. Moreover, six genes annotated as $\beta$-arrestin were also highly expressed in the $\mathrm{VP}_{\mathrm{AHPND}}$-susceptible families.

\section{Gene Set Enrichment Analysis}

By GSEA of GO gene set, there were a total of 265 significantly enriched gene sets between resistant and susceptible groups (nominal $p$-value $<0.05$ and FDR $<0.25$ ). The top 10 gene sets enriched in resistant families were all involved in energy metabolism process, while most gene sets enriched in the 
TABLE 5 | Immunity-related DEGs with high expression in $\mathrm{VP}_{\text {AHPND}}$-susceptible Litopenaeus vannamei families.

\begin{tabular}{|c|c|c|}
\hline Gene ID & Description & Species \\
\hline ncbi_113817858 & C-type lectin domain family 4 member E-like isoform X3 & Lates calcarifer \\
\hline ncbi_113825958 & Serpin B6-like isoform X2 & Sarcophilus harrisii \\
\hline ncbi_113828431 & Serine protease 42 -like & Chrysochloris asiatica \\
\hline ncbi_113808061 & $\alpha$-2-Macroglobulin-like protein 1 & Pseudopodoces humilis \\
\hline ncbi_113808062 & $\alpha$-2-Macroglobulin-like protein 1 & Coturnix japonica \\
\hline ncbi_113816695 & DBH-like monooxygenase protein 1 & Otolemur garnettii \\
\hline ncbi_113804592 & DBH-like monooxygenase protein 1 & O. garnettii \\
\hline ncbi_113813557 & Cytochrome b5-like & Salmo salar \\
\hline ncbi_113807689 & Cytochrome P450 & Danio rerio \\
\hline ncbi_113829525 & $\beta$-Arrestin-2 isoform $\times 2$ & Heterocephalus glaber \\
\hline ncbi_113826122 & $\beta$-Arrestin-2 & Pelodiscus sinensis \\
\hline ncbi_113815019 & $\beta$-Arrestin-2 & Lepisosteus oculatus \\
\hline ncbi_113822594 & $\beta$-Arrestin-1-like, partial & Takifugu rubripes \\
\hline ncbi_113804603 & $\beta$-Arrestin-1, partial & Kryptolebias marmoratus \\
\hline ncbi_113807566 & $\beta$-Arrestin-1 & Gekko japonicus \\
\hline
\end{tabular}

Note. DEGs, differentially expressed genes.

\begin{tabular}{|c|c|c|c|c|c|}
\hline EP & Gene set & Size & NES & Nom $p$-val & FDR \\
\hline $\mathrm{R}$ & Mitochondrial_protein_complex & 78 & 2.70 & 0 & 0 \\
\hline $\mathrm{R}$ & Inner_mitochondrial_membrane_protein_complex & 36 & 2.64 & 0 & 0 \\
\hline $\mathrm{R}$ & Cellular_respiration & 64 & 2.61 & 0 & 0 \\
\hline $\mathrm{R}$ & Oxidative_phosphorylation & 44 & 2.58 & 0 & 0 \\
\hline $\mathrm{R}$ & Mitochondrial_respiratory_chain_complex_assembly & 33 & 2.57 & 0 & 0 \\
\hline $\mathrm{R}$ & Respiratory_electron_transport_chain & 40 & 2.54 & 0 & 0 \\
\hline $\mathrm{R}$ & Respiratory_chain_complex & 27 & 2.53 & 0 & 0 \\
\hline $\mathrm{R}$ & ATP_synthesis_coupled_electron_transport & 35 & 2.52 & 0 & 0 \\
\hline $\mathrm{R}$ & NADH_dehydrogenase_complex_assembly & 26 & 2.49 & 0 & 0 \\
\hline $\mathrm{R}$ & Respirasome & 35 & 2.44 & 0 & 0 \\
\hline S & G_Protein_coupled_receptor_activity & 52 & -2.16 & 0 & 0.119 \\
\hline S & G_Protein_coupled_receptor_signaling_pathway & 115 & -2.11 & 0 & 0.130 \\
\hline$S$ & Tight_junction & 18 & -2.10 & 0 & 0.101 \\
\hline S & Positive_regulation_of_blood_circulation & 8 & -2.10 & 0 & 0.078 \\
\hline S & Solute_sodium_symporter_activity & 32 & -2.10 & 0 & 0.066 \\
\hline S & Neurotransmitter_binding & 17 & -2.10 & 0 & 0.055 \\
\hline S & Neuromuscular_process_controlling_balance & 10 & -2.09 & 0 & 0.048 \\
\hline S & Apical_junction_complex & 18 & -2.05 & 0.003 & 0.072 \\
\hline S & Serine_type_endopeptidase_inhibitor_activity & 17 & -2.05 & 0.003 & 0.069 \\
\hline S & Pattern_recognition_receptor_signaling_pathway & 27 & -2.02 & 0 & 0.085 \\
\hline
\end{tabular}

Note. EP, enrichment in phenotype, gene sets enriched in nine resistant (R) samples or nine susceptible (S) samples; Size, number of genes in the gene set; NES, normalized enrichment score; NOM p-value, nominal p-value, the statistical significance of the enrichment score; FDR, false discovery rate; GSEA, Gene set enrichment analysis; GO, Gene Ontology.

susceptible families were related to signal transduction and immunity, such as $G$ protein-coupled receptor activity, G protein-coupled receptor signaling pathway, and pattern recognition receptor signaling pathway (Table 6). Figure 6 shows GSEA of KEGG subset of canonical pathways. The most significantly enriched pathway in resistant families was oxidative phosphorylation (Figure 6A), which was also related to energy metabolism, supporting the results of GO gene sets. The most significantly enriched pathway in the susceptible families was JAK/STAT signaling pathway (Figure 6B), including protein inhibitor of activated STAT, cytokine-inducible SH2containing protein, and tyrosine-protein phosphatase nonreceptor type 11 isoform $\mathrm{X} 2$.

\section{Verification of Candidate Genes in the Descendant Families}

A total of 32 genes were validated in descendant families. For the 16 DEGs shared by three pairwise comparisons, seven genes were verified successfully in the hepatopancreas, and eight genes were verified successfully in the stomach in their offspring (Table 3, $p<0.05)$. For the other 16 DEGs shared by two pairwise comparisons, three genes were successfully verified in hepatopancreas $(p<0.05)$ (Figure 7A), and six genes were verified successfully in the stomach $(p<0.05)$ (Figure $7 \mathbf{B})$. In summary, a total of 19 genes were successfully verified in the hepatopancreas or stomach, including five genes verified successfully in two tissues. 

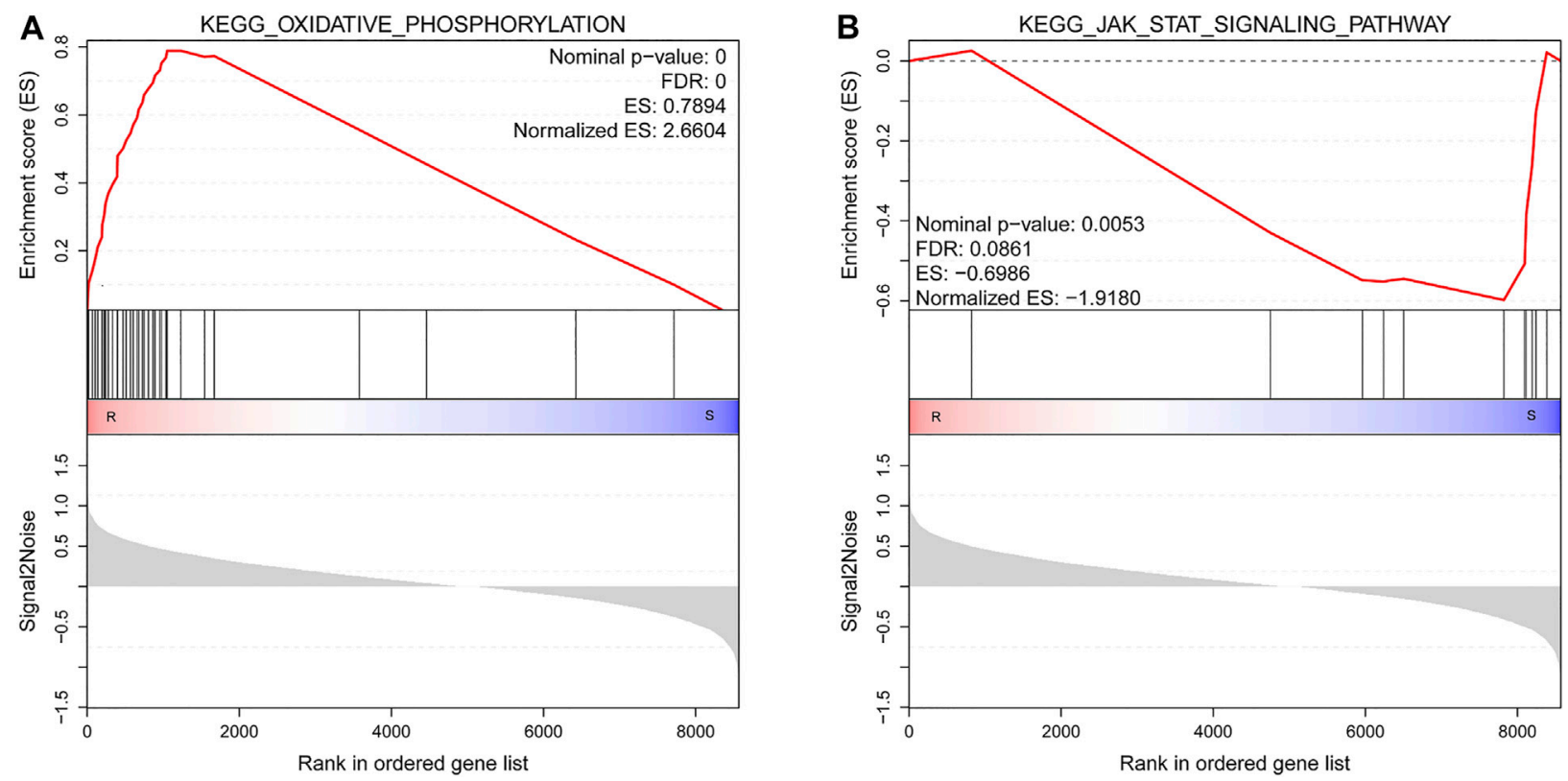

FIGURE 6 | Gene set enrichment analysis (GSEA) of Kyoto Encyclopedia of Genes and Genomes (KEGG) subset of canonical pathways. (A) The most significantly enriched pathway in resistant families. (B) The most significantly enriched pathway in susceptible families. R, resistant samples; S, susceptible samples. Nominal $p$-value, false discovery rate (FDR), enrichment score (ES), and normalized ES were determined by the GSEA software and were indicated within each enrichment plot.

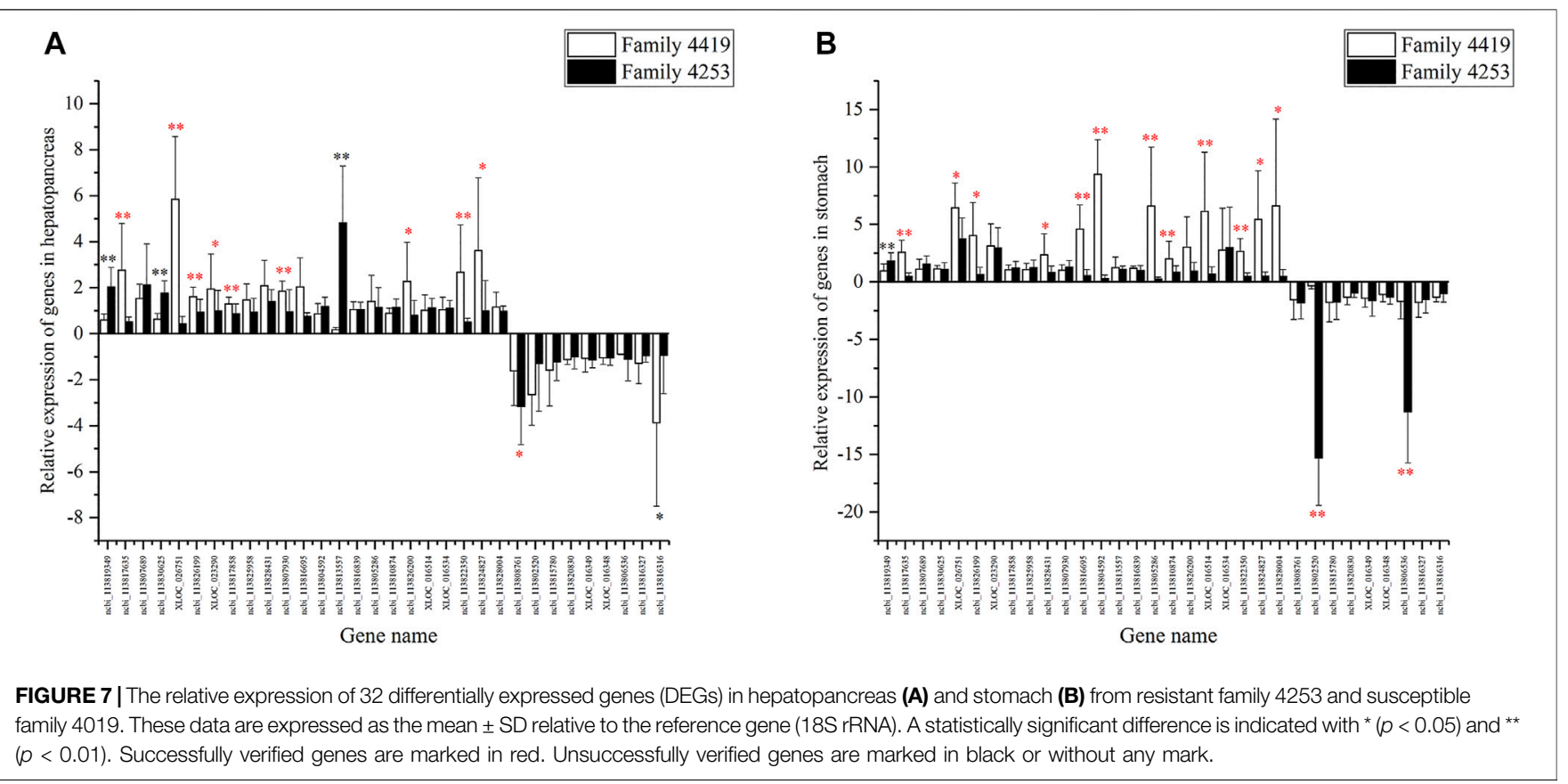

\section{DISCUSSIONS}

It is generally considered that the $\mathrm{VP}_{\mathrm{AHPND}}$ is an opportunistic pathogen. It is pathogenic to cultured shrimp at high concentration or under environmental deterioration condition
(Hong et al., 2016). The physiology and health state of shrimp are closely related to disease resistance. It was estimated that the heritability of the resistance against $\mathrm{VP}_{\mathrm{AHPND}}$ in L. vannamei was near-to-moderate, which indicated that the resistance of shrimp against $\mathrm{VP}_{\mathrm{AHPND}}$ was hereditable (Wang et al., 2019). In this 
study, the resistant and susceptible families of L. vannamei were obtained through continuous challenge test, which provided reliable genetic material for analysis on the disease resistance of shrimp (Grimholt, et al., 2003; Wang et al., 2014). Understanding the gene expression character of diseaseresistant families and the susceptible families could provide useful information for genetic dissection of disease resistance of shrimp.

Recently, an increasing number of transcriptome studies related to AHPND have been performed in L. vannamei, most of which focused on the genes involved in the immune response of shrimp during $\mathrm{VP}_{\mathrm{AHPND}}$ infection (Qi et al., 2017; Maralit et al., 2018; Ng et al., 2018; Qin et al., 2018; Zheng et al., 2018). However, few works have focused on the correlation between the gene expression level and resistance phenotype of shrimp. In this study, we performed RNA-seq analyses to investigate the comparative expression profiles between resistant and susceptible families of shrimp. Gene expression profiles can underlie complex phenotype variations (Crawford and Powers, 1992). The gene expression variation is influenced by various genetic and environmental factors (Leder et al., 2014). Many studies proved that genetic factors influence gene expression in humans (Cheung and Spielman, 2002), mice (Sandberg et al., 2000), Drosophila (Jin et al., 2001), and yeast (Brem et al., 2002). To our knowledge, this is the first report about the comparison of the basal mRNA expression profiles from the omics level of AHPND-resistant and AHPND-susceptible families of $L$. vannamei. The gene expression levels influencing the phenotype variations could also be considered as molecular markers and could be used for genetic selection (Gilad et al., 2008). For aquaculture species, genes related to phenotype variations have been reported. The expression of BIRC7 was correlated with the survival in Vibrio challenge tests in clam $M$. petechialis, and gene expression variation of BIRC7 gene was heritable, indicating the feasibility of selective breeding by reliable genetically based markers (Jiang et al., 2018; Jiang et al., 2019).

In the present study, we found that DEGs highly expressed in the susceptible families of shrimp were mainly related to the immunity, which were in agreement with previous research results. It was reported that the basal expression levels of immune-related genes BIRC7 and NFIL3 were higher in Vibrio-susceptible clams (Jiang et al., 2018). In this study, several genes in proPO activation system showed a higher expression level in the susceptible families. A previous report showed that PPAE2 presented a higher expression level in the susceptible line of L. vannamei after AHPND challenge in comparison with the resistant line (Mai et al., 2021). This system is important for fighting against bacteria pathogens in penaeid shrimp (Amparyup, et al., 2013), and the increased activity of proPO system against Vibrio has been reported in Fenneropenaeus indicus (Sarathi et al., 2007), L. vannamei (Boonchuen et al., 2021), and Macrobrachium rosenbergii (Rao et al., 2015). The C-type lectin was also upregulated in the susceptible families of shrimp; the C-type lectin plays an important role in phagocytosis, melanization, respiratory burst, and coagulation; and it can also activate the proPO system (Junkunlo et al., 2012). Interestingly, C-type lectin 1-like and
Crustin-P had significant higher expression levels in the AHPND-susceptible line of L. vannamei than resistant line at $24 \mathrm{~h}$ post-infection (Mai et al., 2021). In Litopenaeus stylirostris, the basal expression level of antimicrobial peptide was significantly higher in Vibrio-susceptible shrimp line (Lorgeril et al., 2008). In addition, $\beta$-arrestin also played a vital role in the antibacterial immunity of shrimp (Jiang et al., 2013; Sun et al., 2016). The GSEA also illustrated that partial immune-related genes were upregulated in the susceptible families; the JAK/STAT signaling pathway, which was an important signal transduction pathway regulating the immune response in invertebrates, was significantly enriched in the susceptible families (Yu et al., 2017).

Besides, the genes in immunity showed differential expression patterns between susceptible and resistant families; another major part of the DEGs was related to myosin and metabolism. In this study, myosin and many other genes related to metabolism were more active in resistant families. Myosin and actin play a diverse role in a wide range of functions such as cytoskeleton, muscle contraction, and immune response (Marston 2018; Sitbon et al., 2019). It was already reported that myosin light chain was related to the phagocytosis against invading pathogens in Penaeus japonicus, and the transcription level of myosin in WSSVresistant shrimp was nearly two times higher than that in the control shrimp (Han et al., 2010). After pathogen infection, myosin and actin were significantly upregulated in shrimp (Shi et al., 2018; Ren et al., 2019). As for metabolism, we found that enzymes like trypsin, ChyA, PL, and SP and glycolysis pathway including GAPDH and TPI were highly expressed in the resistant families (Table 4). Meanwhile, the top 10 gene sets enriched in resistant families in the GSEA were all involved in energy metabolism process. The finding was consistent with the previous report that SP and ChyB had a significantly higher expression level in resistant shrimp line during the AHPND infection (Mai et al., 2021). Several studies have indicated that metabolic processes such as lipid metabolism and carbohydrate metabolic process in shrimp were greatly affected during AHPND infection (Velazquez-Lizarraga et al., 2019; Kumar et al., 2021). Taken together, the activated myosin, actin, and energy metabolism might indicate that the shrimp were healthier, which led to higher resistance of shrimp to disease.

After validation, several genes showed the same expression pattern in the offspring of susceptible and resistant families. These genes are possible to be developed as biomarkers for disease resistance of shrimp. It was already reported that gene expression profile could be used as an indicator for disease resistance trait. For example, Bsr-d1 RNA level in susceptible rice strain was twofold higher than that in resistant rice strain, and it has been further proved that inhibiting the expression of bsr- $\mathrm{d} 1$ could increase the rice resistance against blast infection ( $\mathrm{Li}$ et al., 2017). In E. carinicauda, eight immune-related genes were suggested as potential disease-resistant parameters for evaluating the physiological status and disease-resistant capability of shrimp when infected with $\mathrm{VP}_{\mathrm{AHPND}}$ (Ge et al., 2018). In this study, the 19 genes successfully verified in their descendant families were expected to be developed as biomarkers of shrimp resistance against Vibrio. Therefore, apart from sib challenge testing and molecular marker-assisted breeding, the gene expression level of 
these 19 genes could also be used as molecular markers for accelerating the breeding of disease-resistant varieties in $L$. vannamei.

In summary, this study integrated the $\mathrm{VP}_{\mathrm{AHPND}}$-resistance phenotype variation and gene expression profiles to identify the genes related to disease resistance of shrimp. A total of 489 DEGs were identified between the resistant and susceptible families, and they were considered to be associated with the ability of $\mathrm{VP}_{\text {AHPND }}$ resistance in $L$. vannamei. Gene annotation and enrichment analysis revealed that the immune response and energy metabolism could influence resistance of shrimp against $\mathrm{VP}_{\mathrm{AHPND}}$. The obtained data provide a fundamental basis for clarifying the genetic mechanism of resistance to bacterial pathogen, and the identified disease resistance genes of shrimp could accelerate the genetic breeding in shrimp aquaculture.

\section{DATA AVAILABILITY STATEMENT}

The datasets presented in this study can be found in online repositories. The names of the repository/repositories and accession number(s) can be found in the article/Supplementary Material.

\section{REFERENCES}

Amparyup, P., Charoensapsri, W., and Tassanakajon, A. (2013). Prophenoloxidase System and its Role in Shrimp Immune Responses against Major Pathogens. Fish Shellfish Immunol. 34 (4), 990-1001. doi:10.1016/j.fsi.2012.08.019

Argue, B. J., Arce, S. M., Lotz, J. M., and Moss, S. M. (2002). Selective Breeding of Pacific White Shrimp (Litopenaeus vannamei) for Growth and Resistance to Taura Syndrome Virus. Aquaculture 204, 447-460. doi:10.1016/s0044-8486(01) $00830-4$

Arora, S., Steuernagel, B., Gaurav, K., Chandramohan, S., Long, Y., Matny, O., et al. (2019). Resistance Gene Cloning from a Wild Crop Relative by Sequence Capture and Association Genetics. Nat. Biotechnol. 37 (2), 139-143. doi:10.1038/s41587-018-0007-9

Boonchuen, P., Jaree, P., Somboonviwat, K., and Somboonwiwat, K. (2021). Regulation of Shrimp Prophenoloxidase Activating System by Lva-miR-4850 during Bacterial Infection. Sci. Rep. 11 (1), 3821. doi:10.1038/s41598-021$82881-2$

Brem, R. B., Yvert, G., Clinton, R., and Kruglyak, L. (2002). Genetic Dissection of Transcriptional Regulation in Budding Yeast. Science 296 (5568), 752-755. doi:10.1126/science.1069516

Cheung, V. G., and Spielman, R. S. (2002). The Genetics of Variation in Gene Expression. Nat. Genet. 32, 522-525. doi:10.1038/ng1036

Crawford, D. L., and Powers, D. A. (1992). Evolutionary Adaptation to Different thermal Environments via Transcriptional Regulation. Mol. Biol. Evol. 9, 806-813. doi:10.1093/oxfordjournals.molbev.a040762

de Lorgeril, J., Gueguen, Y., Goarant, C., Goyard, E., Mugnier, C., Fievet, J., et al. (2008). A Relationship between Antimicrobial Peptide Gene Expression and Capacity of a Selected Shrimp Line to Survive a Vibrio Infection. Mol. Immunol. 45 (12), 3438-3445. doi:10.1016/j.molimm.2008.04.002

Eanes, W. F. (2011). Molecular Population Genetics and Selection in the Glycolytic Pathway. J. Exp. Biol. 214, 165-171. doi:10.1242/jeb.046458

Ge, Q., Li, J., Li, J., Wang, J., and Li, Z. (2018). Immune Response of Exopalaemon carinicauda Infected with an AHPND-Causing Strain of Vibrio parahaemolyticus. Fish Shellish Immunol. 74, 223-234. doi:10.1016/ j.fsi.2017.12.042

Gilad, Y., Rifkin, S. A., and Pritchard, J. K. (2008). Revealing the Architecture of Gene Regulation: the Promise of eQTL Studies. Trends Genet. 24 (8), 408-415. doi:10.1016/j.tig.2008.06.001

\section{AUTHOR CONTRIBUTIONS}

QZ, YY, and FL contributed to the conception and design of the study. ZL performed the statistical analysis. QZ wrote the first draft of the article. QZ, YY, JX, and FL wrote sections of the article. All authors contributed to article revision and read and approved the submitted version.

\section{FUNDING}

This work is supported by the key program of the National Natural Science Foundation of China (31830100), National Key R\&D Program of China (2018YFD0901301, 2018YFD0900103), and China Agriculture Research System-48.

\section{SUPPLEMENTARY MATERIAL}

The Supplementary Material for this article can be found online at: https://www.frontiersin.org/articles/10.3389/fgene.2021.772442/ full\#supplementary-material

Grimholt, U., Larsen, S., Nordmo, R., Midtlyng, P., Kjoeglum, S., Storset, A., et al (2003). MHC Polymorphism and Disease Resistance in Atlantic salmon (Salmo salar); Facing Pathogens with Single Expressed Major Histocompatibility Class I and Class II Loci. Immunogenetics 55 (4), 210-219. doi:10.1007/s00251-0030567-8

Han, F., Wang, Z., and Wang, X. (2010). Characterization of Myosin Light Chain in

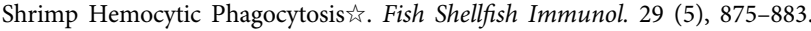
doi:10.1016/j.fsi.2010.07.030

Hong, X. P., Xu, D., Zhuo, Y., Liu, H. Q., and Lu, L. Q. (2016). Identification and Pathogenicity of Vibrio Parahaemolyticus Isolates and Immune Responses of Penaeus (Litopenaeus) vannamei (Boone). J. Fish. Dis. 39 (9), 1085-1097. doi:10.1111/jfd.12441

Houston, R. D., Haley, C. S., Hamilton, A., Guy, D. R., Tinch, A. E., Taggart, J. B., et al. (2008). Major Quantitative Trait Loci Affect Resistance to Infectious Pancreatic Necrosis in Atlantic salmon (Salmo salar). Genetics 178 (2), 1109-1115. doi:10.1534/genetics.107.082974

Huang, Y., Yin, Z., Weng, S., He, J., and Li, S. (2012). Selective Breeding and Preliminary Commercial Performance of Penaeus vannamei for Resistance to white Spot Syndrome Virus (WSSV). Aquaculture 364-365, 111-117. doi:10.1016/j.aquaculture.2012.08.002

Jiang, D., Xie, T., Liang, J., and Noble, P. W. (2013). $\beta$-Arrestins in the Immune System. Prog. Mol. Biol. Transl Sci. 118, 359-393. doi:10.1016/b978-0-12394440-5.00014-0

Jiang, F., Wang, H., Yue, X., Zhang, S., and Liu, B. (2018). Integrating the Vibrio -resistance Phenotype and Gene Expression Data for Discovery of Markers Used for Resistance Evaluation in the Clam Meretrix petechialis. Aquaculture 482, 130-136. doi:10.1016/j.aquaculture.2017.09.033

Jiang, F., Yue, X., Zhang, S., Yu, J., Wang, R., Liu, B., et al. (2019). Heritability of ResistanceRelated Gene Expression Traits and Their Correlation with Body Size of Clam Meretrix petechialis. J. Ocean. Limnol. 38 (2), 571-578. doi:10.1007/s00343-019-8326-3

Jin, W., Riley, R. M., Wolfinger, R. D., White, K. P., Passador-Gurgel, G., and Gibson, G. (2001). The Contributions of Sex, Genotype and Age to Transcriptional Variance in Drosophila melanogaster. Nat. Genet. 29 (4), 389-395. doi:10.1038/ng766

Junkunlo, K., Prachumwat, A., Tangprasittipap, A., Senapin, S., Borwornpinyo, S., Flegel, T. W., et al. (2012). A Novel Lectin Domain-Containing Protein (LvCTLD) Associated with Response of the Whiteleg Shrimp Penaeus (Litopenaeus) vannamei to Yellow Head Virus (YHV). Dev. Comp. Immunol. 37, 334-341. doi:10.1016/j.dci.2011.12.010 
Kim, D., Pertea, G., Trapnell, C., Pimentel, H., Kelley, R., and Salzberg, S. L. (2013). TopHat2: Accurate Alignment of Transcriptomes in the Presence of Insertions, Deletions and Gene Fusions. Genome Biol. 14 (4), R36. doi:10.1186/gb-201314-4-r36

Kumar, R., Tung, T.-C., Ng, T. H., Chang, C.-C., Chen, Y.-L., Chen, Y.-M., et al. (2021). Metabolic Alterations in Shrimp Stomach during Acute Hepatopancreatic Necrosis Disease and Effects of Taurocholate on Vibrio parahaemolyticus. Front. Microbiol. 12, 631468. doi:10.3389/fmicb.2021.631468

Leder, E. H., McCairns, R. J. S., Leinonen, T., Cano, J. M., Viitaniemi, H. M., Nikinmaa, M., et al. (2014). The Evolution and Adaptive Potential of Transcriptional Variation in Sticklebacks-Signatures of Selection and Widespread Heritability. Mol. Biol. Evol. 32, 674-689. doi:10.1093/molbev/ msu328

Lee, C.-T., Chen, I.-T., Yang, Y.-T., Ko, T.-P., Huang, Y.-T., Huang, J.-Y., et al. (2015). The Opportunistic marine pathogen Vibrio parahaemolyticus becomes Virulent by Acquiring a Plasmid that Expresses a Deadly Toxin. Proc. Natl. Acad. Sci. USA 112 (34), 10798-10803. doi:10.1073/pnas.1503129112

Li, W., Zhu, Z., Chern, M., Yin, J., Yang, C., Ran, L., et al. (2017). A Natural Allele of a Transcription Factor in rice Confers Broad-Spectrum Blast Resistance. Cell 170 (1), 114-126. doi:10.1016/j.cell.2017.06.008

Liu, J., Yu, Y., Li, F., Zhang, X., and Xiang, J. (2014a). A New ALF from Litopenaeus vannamei and its SNPs Related to WSSV Resistance. Chin. J. Ocean. Limnol. 32 (6), 1232-1247. doi:10.1007/s00343-015-4010-4

Liu, J., Yu, Y., Li, F., Zhang, X., and Xiang, J. (2014b). A New Antilipopolysaccharide Factor (ALF) Gene with its SNP Polymorphisms Related to WSSV-Resistance of Litopenae vannamei. Fish Shellfish Immunol. 39 (1), 24-33. doi:10.1016/j.fsi.2014.04.009

Livak, K. J., and Schmittgen, T. D. (2001). Analysis of Relative Gene Expression Data Using Real-Time Quantitative PCR and the $2^{-\Delta \Delta}$ CT Method. Methods 25 (4), 402-408. doi:10.1006/meth.2001.1262

Mai, H. N., Caro, L. F. A., Cruz-Flores, R., White, B. N., and Dhar, A. K. (2021). Differentially Expressed Genes in Hepatopancreas of Acute Hepatopancreatic Necrosis Disease Tolerant and Susceptible Shrimp (Penaeus vannamei). Front. Immunol. 12, 634152. doi:10.3389/fimmu.2021.634152

Maralit, B. A., Jaree, P., Boonchuen, P., Tassanakajon, A., and Somboonwiwat, K. (2018). Differentially Expressed Genes in Hemocytes of Litopenaeus vannamei Challenged with Vibrio parahaemolyticus AHPND ( $\left.\mathrm{VP}_{\mathrm{AHPND}}\right)$ and $\mathrm{VP}_{\mathrm{AHPND}}$ Toxin. Fish Shellfish Immunol. 81, 284-296. doi:10.1016/j.fsi.2018.06.054

Marston, S. (2018). The Molecular Mechanisms of Mutations in Actin and Myosin that Cause Inherited Myopathy. Ijms 19 (7), 2020. doi:10.3390/ijms19072020

Moen, T., Baranski, M., Sonesson, A. K., and Kjøglum, S. (2009). Confirmation and fine-mapping of a Major QTL for Resistance to Infectious Pancreatic Necrosis in Atlantic salmon (Salmo salar): Population-Level Associations between Markers and Trait. BMC Genomics 10 (1), 368. doi:10.1186/1471-2164-10-368

Ng, T. H., Lu, C. W., Lin, S. S., Chang, C. C., Tran, L. H., Chang, W. C., et al. (2018). The Rho Signalling Pathway Mediates the Pathogenicity of AHPND-causing $V$. parahaemolyticus in Shrimp. Cell Microbiol. 20 (8), 12. doi:10.1111/cmi.12849

Qi, C., Wang, L., Liu, M., Jiang, K., Wang, M., Zhao, W., et al. (2017). Transcriptomic and Morphological Analyses of Litopenaeus vannamei Intestinal Barrier in Response to Vibrio paraheamolyticus Infection Reveals Immune Response Signatures and Structural Disruption. Fish Shellfish Immunol. 70, 437-450. doi:10.1016/j.fsi.2017.09.004

Qin, Z., Babu, V. S., Wan, Q., Zhou, M., Liang, R., Muhammad, A., et al. (2018). Transcriptome Analysis of Pacific White Shrimp (Litopenaeus vannamei) Challenged by Vibrio parahaemolyticus Reveals Unique Immune-Related Genes. Fish Shellfish Immunol. 77, 164-174. doi:10.1016/j.fsi.2018.03.030

Rao, R., Bing Zhu, Y., Alinejad, T., Tiruvayipati, S., Lin Thong, K., Wang, J., et al. (2015). RNA-seq Analysis of Macrobrachium rosenbergii Hepatopancreas in Response to Vibrio parahaemolyticus Infection. Gut Pathog. 7 (1), 6. doi:10.1186/s13099-015-0052-6

Ren, X., Zhang, Y., Liu, P., and Li, J. (2019). Comparative Proteomic Investigation of Marsupenaeus japonicus Hepatopancreas Challenged with Vibrio parahaemolyticus and White Spot Syndrome Virus. Fish Shellfish Immunol. 93, 851-862. doi:10.1016/j.fsi.2019.08.039

Sandberg, R., Yasuda, R., Pankratz, D. G., Carter, T. A., Del Rio, J. A., Wodicka, L., et al. (2000). Regional and Strain-specific Gene Expression Mapping in the Adult Mouse Brain. Proc. Natl. Acad. Sci. 97 (20), 11038-11043. doi:10.1073/ pnas. 97.20 .11038
Santos, C. A., Andrade, S. C. S., Teixeira, A. K., Farias, F., Guerrelhas, A. C., Rocha, J. L., et al. (2021). Transcriptome Differential Expression Analysis Reveals the Activated Genes in Litopenaeus vannamei Shrimp Families of superior Growth Performance. Aquaculture 531, 735871. doi:10.1016/j.aquaculture.2020.735871

Santos, C. A., Andrade, S. C. S., Teixeira, A. K., Farias, F., Kurkjian, K., Guerrelhas, A. C., et al. (2018). Litopenaeus vannamei Transcriptome Profile of Populations Evaluated for Growth Performance and Exposed to White Spot Syndrome Virus (WSSV). Front. Genet. 9, 120. doi:10.3389/fgene.2018.00120

Sarathi, M., Ahmed, V. P. I., Venkatesan, C., Balasubramanian, G., Prabavathy, J., and Hameed, A. S. S. (2007). Comparative Study on Immune Response of Fenneropenaeus indicus to Vibrio alginolyticus and White Spot Syndrome Virus. Aquaculture 271, 8-20. doi:10.1016/j.aquaculture.2007.07.002

Shi, X., Meng, X., Kong, J., Luan, S., Luo, K., Cao, B., et al. (2018). Transcriptome Analysis of 'Huanghai No. 2' Fenneropenaeus chinensis Response to WSSV Using RNA-Seq. Fish Shellfish Immunol. 75, 132-138. doi:10.1016/ j.fsi.2018.01.045

Sitbon, Y. H., Yadav, S., Kazmierczak, K., and Szczesna-Cordary, D. (2019). Insights into Myosin Regulatory and Essential Light Chains: a Focus on Their Roles in Cardiac and Skeletal Muscle Function, Development and Disease. J. Muscle Res. Cel Motil 41, 313-327. doi:10.1007/s10974-019-09517-x

Soonthornchai, W., Chaiyapechara, S., Klinbunga, S., Thongda, W., Tangphatsornruang, S., Yoocha, T., et al. (2016). Differentially Expressed Transcripts in Stomach of Penaeus monodon in Response to AHPND Infection. Dev. Comp. Immunol. 65, 53-63. doi:10.1016/ j.dci.2016.06.013

Subramanian, A., Tamayo, P., Mootha, V. K., Mukherjee, S., Ebert, B. L., Gillette, M. A., et al. (2005). Gene Set Enrichment Analysis: a Knowledge-Based Approach for Interpreting Genome-wide Expression Profiles. Proc. Natl. Acad. Sci. 102 (43), 15545-15550. doi:10.1073/pnas.0506580102

Sun, J.-J., Lan, J.-F., Shi, X.-Z., Yang, M.-C., Niu, G.-J., Ding, D., et al. (2016). $\beta$-Arrestins Negatively Regulate the Toll Pathway in Shrimp by Preventing Dorsal Translocation and Inhibiting Dorsal Transcriptional Activity. J. Biol. Chem. 291 (14), 7488-7504. doi:10.1074/jbc.M115.698134

Trapnell, C., Roberts, A., Goff, L., Pertea, G., Kim, D., Kelley, D. R., et al. (2012). Differential Gene and Transcript Expression Analysis of RNA-Seq Experiments with TopHat and Cufflinks. Nat. Protoc. 7 (3), 562-578. doi:10.1038/ nprot.2012.016

Velázquez-Lizárraga, A. E., Juárez-Morales, J. L., Racotta, I. S., VillarrealColmenares, H., Valdes-Lopez, O., Luna-González, A., et al. (2019). Transcriptomic Analysis of Pacific White Shrimp (Litopenaeus vannamei, Boone 1931) in Response to Acute Hepatopancreatic Necrosis Disease Caused by Vibrio parahaemolyticus. PLoS ONE 14 (8), e0220993. doi:10.1371/journal.pone.0220993

Wang, L., Fan, C., Liu, Y., Zhang, Y., Liu, S., Sun, D., et al. (2014). A Genome Scan for Quantitative Trait Loci Associated with Vibrio anguillarum Infection Resistance in Japanese Flounder (Paralichthys olivaceus) by Bulked Segregant Analysis. Mar. Biotechnol. 16 (5), 513-521. doi:10.1007/s10126-014-9569-9

Wang, P.-H., Wan, D.-H., Gu, Z.-H., Deng, X.-X., Weng, S.-P., Yu, X.-Q., et al. (2011). Litopenaeus vannamei Tumor Necrosis Factor ReceptorAssociated Factor 6 (TRAF6) Responds to Vibrio alginolyticus and White Spot Syndrome Virus (WSSV) Infection and Activates Antimicrobial Peptide Genes. Dev. Comp. Immunol. 35 (1), 105-114. doi:10.1016/ j.dci.2010.08.013

Wang, Q., Yu, Y., Zhang, Q., Zhang, X., Huang, H., Xiang, J., et al. (2019). Evaluation on the Genomic Selection in Litopenaeus vannamei for the Resistance against Vibrio parahaemolyticus. Aquaculture 505, 212-216. doi:10.1016/j.aquaculture.2019.02.055

Woldemariam, N. T., Agafonov, O., Sindre, H., Høyheim, B., Houston, R. D., Robledo, D., et al. (2020). miRNAs Predicted to Regulate Host Anti-viral Gene Pathways in IPNV-Challenged Atlantic salmon Fry Are Affected by Viral Load, and Associated with the Major IPN Resistance QTL Genotypes in Late Infection. Front. Immunol. 11, 2113. doi:10.3389/fimmu.2020.02113

Yu, Y., Liu, J., Li, F., Zhang, X., Zhang, C., and Xiang, J. (2017). Gene Set Based Association Analyses for the WSSV Resistance of Pacific White Shrimp Litopenaeus vannamei. Sci. Rep. 7, 40549. doi:10.1038/srep40549

Zhang, Q., Yu, Y., Wang, Q., Liu, F., Luo, Z., Zhang, C., et al. (2019). Identification of Single Nucleotide Polymorphisms Related to the Resistance against Acute Hepatopancreatic Necrosis Disease in the Pacific White Shrimp Litopenaeus 
vannamei by Target Sequencing Approach. Front. Genet. 10, 11. doi:10.3389/ fgene.2019.00700

Zhang, X., Yuan, J., Sun, Y., Li, S., Gao, Y., Yu, Y., et al. (2019). Penaeid Shrimp Genome Provides Insights into Benthic Adaptation and Frequent Molting. Nat. Commun. 10 (1), 356. doi:10.1038/s41467-018-08197-4

Zheng, Z., Wang, F., Aweya, J. J., Li, R., Yao, D., Zhong, M., et al. (2018). Comparative Transcriptomic Analysis of Shrimp Hemocytes in Response to Acute Hepatopancreas Necrosis Disease (AHPND) Causing Vibrio parahemolyticus Infection. Fish Shellfish Immunol. 74, 10-18. doi:10.1016/j.fsi.2017.12.032

Conflict of Interest: The authors declare that the research was conducted in the absence of any commercial or financial relationships that could be construed as a potential conflict of interest.
Publisher's Note: All claims expressed in this article are solely those of the authors and do not necessarily represent those of their affiliated organizations, or those of the publisher, the editors, and the reviewers. Any product that may be evaluated in this article, or claim that may be made by its manufacturer, is not guaranteed or endorsed by the publisher.

Copyright ( 2021 Zhang, Yu, Luo, Xiang and Li. This is an open-access article distributed under the terms of the Creative Commons Attribution License (CC BY). The use, distribution or reproduction in other forums is permitted, provided the original author(s) and the copyright owner(s) are credited and that the original publication in this journal is cited, in accordance with accepted academic practice. No use, distribution or reproduction is permitted which does not comply with these terms. 